Elsevier Editorial System(tm) for Journal of Chromatography A Manuscript Draft

Manuscript Number: JCA-08-1320R2

Title: Accuracy and precision in determination of perfluorinated chemicals in human blood verified by interlaboratory comparisons

Article Type: REACH 2008

Keywords: PFCs; interlaboratory comparison; QA/QC; human blood; LC/MS; LC/MS/MS

Corresponding Author: Dr. Gunilla Lindström,

Corresponding Author's Institution: Örebro University

First Author: Gunilla Lindström

Order of Authors: Gunilla Lindström; Anna Kärrman; Bert van Bavel 
Elsevier Editorial System(tm) for Journal of Chromatography A

Manuscript Draft

Manuscript Number: JCA-08-1320

Title: Accuracy and precision in the determination of perfluorinated chemicals in human blood verified by interlaboratory comparisons

Article Type: REACH 2008

Keywords: PFCs; interlaboratory comparison; QA/QC; human blood; LC/MS; LC/MS/MS

Corresponding Author: Dr. Gunilla Lindström,

Corresponding Author's Institution: Örebro University

First Author: Gunilla Lindström

Order of Authors: Gunilla Lindström; Anna Kärrman; Bert van Bavel 


\title{
Accuracy and precision in the determination of perfluorinated chemicals in human blood verified by interlaboratory comparisons
}

\author{
Gunilla Lindström*, Anna Kärrman and Bert van Bavel \\ Man-Technology-Environment Research Centre (MTM) \\ Örebro University \\ SE-701 82 Örebro \\ Sweden \\ Tel. +46 19301098 \\ gunilla.lindstrom@nat.oru.se
}

Keywords: perfluorinated chemicals, PFOS, PFOA, analysis, QA-QC

\begin{abstract}
Perfluorinated chemicals, PFCs, are analyzed in laboratories worldwide to determine human blood levels and exposure pathways. The development of the analytical technique has been rapid in the last ten years, and prerequisites for accurate and precise determination of PFCs in human blood at low $\mathrm{ng} / \mathrm{g}$ concentrations are today readily available. The main contributing factors are the improved LC-MS instrumentations, the increased availability of native and mass labeled PFC standards, and new column materials available for chromatographic separations. The results of the first international interlaboratory study (ILS) in 2005 on PFCs revealed relatively better analytical results for human blood analyses when compared to analyses of a number of environmental matrices. The representative accuracy for the analyses of PFCs in human matrixes reported in recent years was established in the second human serum ILS in 2006. Interlaboratory standard deviations for the two human serum samples one low level concentration and one medium level concentration were found to be $12 \%$ and $16 \%$ for PFOS, respectively, and $47 \%$ and $21 \%$ for PFOA, respectively. Reported detections for all PFCs followed a frequency of $\mathrm{PFOS}>\mathrm{PFOA}>\mathrm{PFHxS}>\mathrm{PFNA}>\mathrm{PFDA}>>\mathrm{PFDoA}>>$ PFDS $>>$ PFHxA. Due to the small number of reported values for the other perfluorosulfonates and perfluorocarboxylates, standard deviations were not established.
\end{abstract}




\section{Introduction}

Since 2000 an increasing number of reports have confirmed the world-wide occurrence of perfluorinated chemicals, PFCs, in human blood. The growing demand for monitoring and exposure assessment to humans and regulation of this group of omnipresent environmental pollutants has led to the rapid development and refinement of the analytical chemical procedures. The problems concerning the reliability of PFC analysis in their early stage were discussed at an international workshop organized in Germany in 2003 followed by a joint paper by Martin et al. in 2004 [1]. The lack of certified reference materials, interlaboratory comparison studies, and native as well as labeled standards were found to hamper PFC research and standardization. Many of the problems have since been successfully addressed and the qualitative and quantitative determination of PCFs in blood has been evaluated in a number of recent method development and interlaboratory studies. These studies showed that the analytical prerequisites for accurate, precise and reproducible PFC data to a large extent are met today.

The first worldwide PFC interlaboratory comparison study on human plasma and whole blood, in addition to a number of other environmental matrixes, was organized in 2005 by The Netherlands Institute for Fisheries Research (RIVO), IJmuiden, the Netherlands and the MTM Research Institute at the Örebro University, Örebro, Sweden [2]. The objective was to determine the current level of interlaboratory agreement between the determinations of different PFCs in various matrixes. For the human matrixes, unfortified plasma and blood were used, and each laboratory used their in-house method. The target PFCs were perfluorobutane sulfonate (PFBS), perfluorohexane sulfonate (PFHxS), perfluorooctane sulfonate (PFOS), perfluorodecanoic acid (PFDA), perfluorohexanoic acid (PFHxA), perfluoroheptanoic acid (PFHpA), perfluorononaoic acid (PFNA), perfluorooctanoic acid (PFOA), perfluorodecane sulfonate (PFDS) perfluoroundecanoic acid (PFUnA), perfluorododecanoic acid (PFDoA), perfluorotetradecanoic acid (PFDA), perfluorooctanesulfonamide (PFOSA)

Seventeen laboratories submitted results for the human matrixes. The results showed the best agreement among the laboratories for determination of PFOS and PFOA in the human matrixes when compared to the environmental ones.

The second worldwide interlaboratory study on PFCs in human serum was performed in 2006. In this second study, two standard reference materials, Human Serum SRM 1589a and1957 from NIST, were used. Three ${ }_{13} \mathrm{C}$ labeled standards (PFOS, PFOA and PFNA) weremade available in addition to the ${ }_{12} \mathrm{C}$ study standard which contained eight $\mathrm{PFCs}$ (PFHx, PFOA, PFNA, PFDA, PFDoA, PFHxS, PFOS and PFDS). Fifteen laboratories, several of which had participated in the earlier interlaboratory study, took part and submitted results. Agreement among the participating laboratories' results for the serum, plasma, and whole blood samples and PFOS and PFOA standards in this second interlaboratory study when compared to the first confirmed that the development and refinement of analytical techniques applied in PFC analyses improved the performance of these techniques. 


\section{Analytical methodology}

The analytical methodologies for determination of PFCs have gone through a rapid development since the 1980s. The main contributing factors for today's considerably improved analytical performance for qualitative and quantitative determination of PFCs are the improved LC-MS instrumentations, the increased availability of native and mass labeled PFC standards and new materials available for chromatographic separations. In addition to this the increasing analytical experience due to a growing demand for data on PFC world-wide has had a distinct effect on the enhancement of the quality.

Sample preparation - The first breakthrough in the field of PFC determination in human samples was the ion-pair extraction and LC/MS/MS method published by Hansen et al. in 2001 [3]. The ion-pairing extraction using tetrabutylammonium salt and methyl tert-butyl ether was initially developed by Ylinen $\underline{e t}$ al. in 1985 [4]. Later, published methods for human matrices typically involve protein precipitation, using acid or acetonitrile followed by direct analysis [5] or additional clean-up with solid-phase extraction (SPE) [6-8], or dispersive carbon clean-up [9].

Approximately one-third of the 19 laboratories participating in the human plasma and human whole blood part of the first worldwide ILS in 2005 used the ion-pairing method [2], although it lacks a clean-up step and is thus sensitive to co-extracted interferences. Approximately one third used solid-phase extraction and the remaining third acetonitrile precipitation without further clean-up. A more specific SPE adsorbent especially for the fluorinated carboxylates and sulfonates became commercially available in 2004. This adsorbent, a weak ion exchange (WAX), proved to be well suited for a variety of sample types by Taniyasu in 2005 [8] and Kärrman in 2007 [14] and exhibits very specific adsorption properties for acids such as PFOS and PFOA. The extraction and clean-up methods in the second intercalibration used mainly different types of solid-phase extraction systems and columns (Oasis WAX/HLB, Polaris C18, Sep-Pak) and, to lesser extent, ion-pair extraction and acetonitrile precipitation.

Three ${ }_{13} \mathrm{C}$ labeled internal standards for PFOS, PFOA and PFNA (Wellington Laboratories Guelph, Ontario, Canada) were made available in 2004 and were used in the second human matrixes interlaboratory study. This enabled quantification by isotope dilution with enhanced quality assurance as with other POP analysis such as for dioxins, BFRs and PCBs. Today, a sufficient number of labeled as well as isomer specific (linear and branched sulfonates) PFC standards are available for isotope dilution and isomer specific quantification.

Already at the time of the first interlaboratory study in 2005 the laboratories specialized in human matrixes reported on having a higher level of experience $(56 \%$ had $>3$ years experience) than those analyzing the environmental matrixes $(30 \%$ had $>3$ years experience). Of course, this is one of the contributing factors for the better achievement in blood analyses. 
Detection techniques - Non specific methods for the analysis of PFCs were performed in the 1960s by ion selective electrodes or nuclear magnetic resonance. In the 1980s GC based methods were developed for the carboxylates including PFOA after derivatisation, performed at the $\mu \mathrm{g} / \mathrm{ml}$ level [4]. Parallel with the development of reliable LC/MS interfaces, including electro spray, sensitive, selective and reliable instrumentation for the analysis of PFCs became available. This enabled the detection at low $\mathrm{ng} / \mathrm{g}$ concentrations of PFOS and PFOA in humans [3].

Presently, the most frequently used detection technique for anionic PFCs in human matrices is negative electrospray triple quadrupole LC/MS/MS [2]. Berger $\underline{\text { et }} \underline{\text { al. }}$ compare ion-trap, time-of-flight (ToF) high resolution MS and triple quadrupole MS as detection techniques for anionic PFCs and fluorotelomer alcohols [11]. While quadrupole MS/MS is the best choice for telomer alcohols, ToF is the technique resulting in highest selectivity and sensitivity for anionic PFCs. A few reports describe extraction and analytical conditions suitable for single quadrupole LC/MS detection of anionic PFCs [7, 12]. Electrospray-MS detection is especially sensitive for signal enhancement or suppression induced by coeluting compounds in the mass spectrometer's ion source. With increasing number of isotope-labeled standards now commercially available the quantification error from matrix effects are reduced and the quality of analysis is improved. Benskin et $\underline{a l}$. emphasize the importance of the LC separation method when reporting taurodeoxycholate isomers interfering with $\mathrm{m} / \mathrm{z} 499>80$ transition of PFOS using a common alkyl stationary phase. Co-eluting endogenous steroid sulfates were also identified causing a 10-20 fold over-reporting of perfluorohexane sulfonate (PFHxS) in human serum [15].

Separation of linear and branched isomers has also been recognized as important in ultratrace analysis of PFCs [13]. Human blood contains PFOS isomers [14] that, if not separated from the linear isomer, introduce quantification error due to different response in the mass spectrometer. Analyses of the neutral PFCs such as PFOSA and its methyl, ethyl derivates and telomer alcohols are more complex. PFOSA and the telomere alcohols can be analyzed both by GC or LC. Good results have been obtained by GC/MS and GC/MS/MS [17 ] but also LC/MS [7]. It is currently unclear which technique will result in the best detection limits. Separation of an ionic and a neutral fraction on WAX SPE cartridges is possible followed by subsequent analysis on LC/MS/MS or GC/MS for the neutral fraction.

Basis for reporting of PFC blood levels - The relatively poorly understood distribution behavior of different PFCs between human whole blood, plasma, and serum needs to be taken into consideration when reporting and comparing results. Frequency distributions between plasma and whole blood were established in a study by Kärrman to be 1.2 for PFOS, 1.4 for PFOA, 1.2 for PFHxS and 0.2 for PFOSA [18]. Average concentrations of PFOS and PFOA in plasma were thus 1.2 and 1.4 times higher than in whole blood and using a general factor of 2 (based on complete PFC binding to plasma/serum proteins) to convert whole blood levels to plasma levels would result in overestimations of the PFOS concentration by about $60 \%$ and of the PFOSA concentration by an order of magnitude. The second PFC interlaboratory study on human blood defines the current state-of-art. This worldwide interlaboratory study organized in 2006 shows results which are 
representative for the analytical performance for PFCs reported in recent years. In the study twenty-one laboratories world-wide took part. Fifteen of the laboratories submitted results. Two NIST SRM serums, Serum A (low level) and Serum B (medium level), were used in the study together with a Study Standard C. The objectives were to assure accuracy and consistency in analytical data from PFC analyses and to support laboratories in their efforts to develop and perform PFC analyses. For laboratories entering the field, exchange of analytical know-how with more experienced laboratories are of great help. For those laboratories seeking documented quality assurance this study serves that purpose.

\section{Experimental}

\section{Study design}

Participating laboratories were asked to, at least, determine the concentrations of PFOS and PFOA in the two human serum samples. The samples were not fortified with PFCs and represented naturally contaminated serum and, therefore, it was expected that concentrations of the other PFCs would be relatively low. All analyses should be performed in triplicate. Optionally, the samples should be analyzed for PFHxA, PFNA, PFDoA, PFHxS and PFDS and the results submitted for evaluation. The participants could use any method they wanted and their own standards and quantification procedures. A short description of the extraction methods and the analytical instrumentation used should be included with the results. Submission of the result forms was performed by means of electronic mail, compiled and reconfirmed by the participant prior to the final statistics.

In the study, two serum samples (Human Serum Sample A and Human Serum Sample B) and one PFC ${ }_{12} \mathrm{C}$ standard solution (Standard Solution C) were distributed to the laboratories. Both serums, $\mathrm{A}$ and $\mathrm{B}$, were freeze-dried human serum and represented 10.0 $\mathrm{mL}$ and $10.7 \mathrm{~mL}$ of reconstituted serum, respectively. In addition, small amounts of ${ }_{13} \mathrm{C}$ labeled standards (PFOS, PFOA and PFNA) were made available for testing and use as internal and recovery standards.

Human Serum Sample A (NIST SRM 1589a) - This sample was a freeze-dried standard reference material (SRM) provided by NIST consisting of 50 pooled blood samples of 5 $\mathrm{ml}$ from donors who consumed fish catched around the Great Lakes. The sample was shown to have low concentrations (PFOS $\sim 5 \mathrm{ng} / \mathrm{mL}$ ) when compared to current human background levels of PFCs as can be seen in the results section in this report. Handling and reconstitution of the serum samples were done by the laboratories before analyses according to detailed instructions included in the shipment.

Human Serum Sample B (NIST SRM 1957) - This sample was another freeze-dried SRM also provided by NIST. This sample was prepared from a serum pool of $200 \mathrm{~L}$ across the US. The sample contained medium concentrations (PFOS $\sim 23 \mathrm{ng} / \mathrm{mL}$ and PFOA $\sim 5$ $\mathrm{ng} / \mathrm{mL}$ ) of PFCs in relation to background levels reported in human serum. Detailed information on both SRMs and certificates of analysis can be found at www.nist.gov 
The Study Standard C (Wellington ${ }^{12} \mathrm{C}$ standard solution) - Standard C contained eight ${ }^{12} \mathrm{C}$ PFCs in the $\mathrm{ng} / \mathrm{mL}$ The concentrations were unknown to the laboratories and should be determined using the laboratories own standards. The standard solution had been prepared at a concentration of $10 \mathrm{ng} / \mathrm{mL}$ for each PFC, and the total volume in each ampoule was approximately $200 \mu \mathrm{L}$ in methanol.

Internal and recovery standards (Wellington ${ }_{13} \mathrm{C}$ mass labeled standard) - The mass labeled standard ampoules provided contained PFOS/PFOA $(13 \mathrm{C} \mathrm{M}+4)$ at $5 \mu \mathrm{g} / \mathrm{mL}$ and PFNA $\left({ }^{13} \mathrm{C} \mathrm{M}+5\right)$ at $5 \mu \mathrm{g} / \mathrm{mL}\{$. The total volumes of these two standard solutions were approximately $200 \mu \mathrm{L}$ in methanol.

\section{Analytical methods used}

A brief description of the sample preparation, extraction, clean-up procedure, handling of final extract, instruments used, type of calibration, quantification, internal standards, recovery standards and other standards used were returned by the participants together with the results. Since all laboratories used their own in-house methods and procedures, there is some differences in the various analytical approaches taken. However, in spite of the differences, the results demonstrated that the precision and accuracy are very consistent for the majority of the participating laboratories.

Extraction and Clean-up methods - The extraction and clean-up methods used included different types of solid-phase extraction systems and columns (Oasis WAX/HLB, Polaris C18 prospect-2, Sep-Pak) and also, to a lesser extent, ion-pair extraction and acetonitrile precipitation. The amount of sample analyzed from the reconstituted serum varied between $100 \mu \mathrm{L}$ and $1 \mathrm{~mL}$, and the final volume for LC injection after extraction and clean-up was typically 5-20 $\mu \mathrm{L}$ for the majority of the laboratories. One laboratory used an on-line SPE-HPLC application and its final volume was $400 \mu \mathrm{L}(100 \mu \mathrm{L}$ serum).

LC and MS methods - Various liquid chromatography and mass spectrometry configurations and instruments were used: LC-ESI-MS/MS (triple quad), LC-ESIMS(/MS) (ion trap), LC ESI-MS (single quad), LC-ESI-TOF-MS. HPLC columns such as Betasil C18, Genesis C18, Discovery C18, Zorbax Eclipse/EDB were used with or without their associated pre-columns. Mobile phases were aqueous solutions of ammonium acetate, methanol and, to a limited extent, acetonitrile and acetic acid. MS negative ionization (electro spray, turbo spray) and SIM or MRM were used by all laboratories.

Quantification methods - Various external and internal standards were used for the final of labeled standards $\left({ }^{13} \mathrm{C}\right.$ and $\left.{ }^{18} \mathrm{O}\right)$ as internal and recovery standards, and some used the three ${ }_{13} \mathrm{C}$ PFC standards provided (PFOA, PFOS as internal and PFNA as recovery standard). Matrix matched calibrations using goat, rabbit, or chicken serum were reported as well as standard addition to the intercalibration samples. The native PFC standards used originated from various suppliers (e.g., Wellington, Fluka, Aldrich, 3M, Oakwood Products, Wako, Fluorochem, Strem, Tokyo Chem) with reported purities of $86-99 \%$. Only one laboratory had separated linear from branched peaks (PFOS, PFOSA) when integrating and quantifying the peaks in the samples as well as in the Standard Solution C. 


\section{Results and discussion}

Agreement among the participating laboratories' serum, plasma, and blood and standard PFOS and PFOA results increased considerably in this interlaboratory study when compared to the study performed in 2005 . However, due to the very low PFC concentrations in Serum A, the RSD (47\%) for PFOA in this sample was similar to the RSD in the first study. All laboratories reported results for PFOS in Serum A (low concentration) and for both PFOS and PFOA in Serum B (medium concentration). Reporting frequency for all PFCs was: PFOS $>$ PFOA $>$ PFHxS $>$ PFNA $>$ PFDA $>>$ PFDoA $>$ PFDS $>>$ PFHxA.

Results for Standard C indicated that it would be advisable for laboratories to check their quantification standards or procedures since only a few laboratories were accurate in determining the PFCs in the provided standard which had a concentration of $10 \mathrm{ng} / \mathrm{mL}$.

The summary [Table 1] tabulated the number of entries (n) used for calculations, with the mean concentrations in $\mathrm{ng} / \mathrm{mL}$ and the $\% \mathrm{RSDs}$ for the PFCs reported by the laboratories. The second [the first column contains the names of the analytes] column presents mean concentrations for all data for the test materials and give the \%RSDs when all entries are included, and the third column gives the \%RSDs when outliers ( $>2 \mathrm{SD}$ ) have been removed.

\section{Sample A Human Serum (NIST SRM 1589a)}

The PFC triplicate analyses are presented with the mean, the \% RSDs and the number of entries (n) used in the calculations of all results. All fifteen laboratories reported concentrations above the detection limits (DLs) for PFOS; eight laboratories found PFOA and PFHxS above DLs; and six laboratories found PFNA above DLs. Even with the overall low PFC concentrations of this serum sample, the SD (14\%) for all reported PFOS levels are considered very good, and also the SD (47\%) for PFOA is satisfactory. Conclusions on SDs should be made with caution due to the relatively low number of detected homologues for most of the PFCs.

As an example, the results for PFOS and PFOA are graphically shown in Figure 1 and 2. The interlaboratory RSD \% have been calculated after removing obvious outliers ( $\mathrm{SD}>2$ ). Non detects or less than values were not included in the calculation of the mean and standard deviation.

\section{Sample B Human Serum (NIST SRM 1957)}

All fifteen laboratories reported levels above DLs for both PFOS and PFOA; fourteen laboratories detected PFHxS above the DLs; and eleven laboratories detected PFNA. In this sample, which had levels corresponding to what is found in populations with current PFC background contamination levels [19], PFDA were detected by seven laboratories. However, the SDs did not improve when compared to the low concentration serum until the outliers were removed. It can be concluded that in serum at this contamination level, most laboratories are able to determine at least PFOS and PFOA with acceptable results. 
As an example, the results for PFOS and PFOA are graphical represented in Figure 3 and 4 along with the interlaboratory \% RSD after removing obvious outliers ( $\mathrm{SD}>2$ ).

\section{Study Standard C}

In the original solution provided by Wellington Laboratories the designated concentrations for all 8 PFCs in Standard C were $10 \mathrm{ng} / \mathrm{mL}$. A considerable variation in the reported concentrations was seen among the congeners. This suggests that some of the laboratories should verify and validate the concentrations of their in-house standards. In addition, it appears that few laboratories have mastered the ability to quantitatively determine the whole set of the PFCs in the standard solution. The RSDs for PFOS and PFOA in the Standard C are presented in Figures 5 and 6.

The consensus RSDs for PFOS for the two serum samples of $12 \%$ and $16 \%$ demonstrate very good precision for this compound in this study. The results for the PFOA, PFNA, PFDA and PFHxS were good for Serum Sample B with RSDs ranging from 20-36\%. The RSDs for the Serum Sample A were somewhat higher for PFNA (36\%) and PFOA (47\%), but extreme for PFHxS (108\%). The consensus results for the standard solution, after omitting the outliers, was acceptable ranging from $20 \%$ to $33 \%$ and in good agreement with the designated values. However the RSDs for all data were somewhat higher than expected and varied between $20-56 \%$.

\section{Z-scores}

Using the consensus values from Table $1, \mathrm{z}$-scores were calculated as $\mathrm{z}=(\mathrm{x}-\mathrm{X}) / \mathrm{SD}$, where $\mathrm{x}$ is the mean value of the results reported by the participant (1-20), $X=$ the consensus value and $\mathrm{SD}=$ the standard deviation of the results of all laboratories. The consensus value was calculated as the mean value reported after omitting outliers outside two times the original RSD. This way a z-score based on the actual submitted data is created depending on the RSD of the data submitted. A value between $-2<z$-score $<2$ is satisfactory, a value between $-3<\mathrm{z}$-score $<-2$ and $2<\mathrm{z}$ score $<3$ is questionable and zscore $<-3$ or $>3$ are unsatisfactory. Z-score have been calculated for PFOS, PFOA, PFNA and PFHxS for which sufficient data was available. As an example a graphical representation of the z-score for serum B for both PFOS and PFOA are given in Figures 7 and 8 .

\section{Conclusions}

Given the prerequisites of appropriate instrumentation, well-adapted sample preparation, access to native and labeled standard compounds and experience laboratories world-wide are today capable of determining the most prevalent PFCs in human blood with accuracy and precision suited to serve the monitoring and exposure assessment of these chemicals..

\section{Acknowledgements}

All laboratories which contributed with data to the interlaboratory evaluation study are kindly acknowledged. Wellington Laboratories is gratefully acknowledged for making standards available as well as NIST for providing the SRMs. 


\section{References}

[1] J.W. Martin, K. Kannan, U. Berger, P. de Voogt, J. Field, J. Franklin, J. P.

Geisy, T. Harner, D. C. G. Muir, B. Scott, M. Kaiser, U. Järnberg, K. C. Jones, S. C.

Mabury, H. Schroeder, M. Simcik, C. Sottani, B. van Bavel, A. Kärrman, G. Lindström, S. van Leeuwen, Environ. Sci. Technol. 38 (2004) 249A.

[2] S. P. J. van Leeuwen, A. Kärrman, B. van Bavel, J. de Boer, G. Lindström, Environ.

Sci. Technol. 40 (2006) 7854.

[3] K. J. Hansen, L. A. Clemen, M. E. Ellefson, H. O. Johnson, Environ. Sci. Technol. 35 (2001) 766.

[4] M.Ylinen, H. Hanhijärvi, P. Peura, O. Rämö, Arch Environ Contam Toxicol 14 (1985) 713.

[5] J. M. Flaherty, P. D. Connolly, E. R. Decker, S. M. Kennedy, M. E. Ellefson, W.

K. Reagen, B. Szostek, J. Chromatogr. B 819 (2005) 329.

[6] Z. Kuklenyik, J. A Reich, L. L. Tully, L. L. Needham, A. M. Calafat, Environ. Sci. Technol. 38 (2004) 3698.

[7] A. Kärrman, B. van Bavel, U. Järnberg, L. Hardell, G. Lindström, Anal Chem 77 (2005) 864.

[8] S. Taniyasu, K. Kannan, M. K. So, A. Gulkowska, E. Sinclair, T. Okazawa, N. Yamashita, J. Chromatogr. A 1093 (2005) 89.

[9] C. R. Powley, S. W. George, T. W Ryan, R. C. Buck, Anal Chem 77 (2005) 6353. [10] Y. Miyake, N.Yamashita, M. K. So, P. Rostkowski, S. Taniyasu, P. K. Lam, K. Kannan, J Chromatogr. A 1154 (2007) 214.

[11] U. Berger, I. Langlois, M. Oehme, R. Kallenborn, Eur J Mass Spec 10 (2004) 579 .

[12] L. Maestri, S. Negri, M. Ferrari, M. Ghittori, F. Fabris, P. Danesino, M. Imbriani, Rapid Commun Mass Spectrom 20 (2006) 2728.

[13] I. Langlois, M. Oehme, Rapid Commun Mass Spectrom 20 (2006) 844.

[14] A. Kärrman, I. Langlois, B. van Bavel, G. Lindström, M. Oehme, Environ. Int. 33 (2007) 782.

[15] J. P. Benskin, M. Bataineh, J. W. Martin, Anal Chem 79 (2007) 6455.

[16] S.A., Tittlemier, K. Pepper, L. Edwards, J Agric Food Chem. 54 (2006) 8385.

[17] I. Ericson, K. Worrall, B. van Bavel, T. Takasuga, G. Lindström,

Organohalogen Compds. 69 (2007) 990.371 [

[18] A. Kärrman, B. van Bavel, U. Järnberg, L. Hardell, G. Lindström, Chemosphere 54 (2006) 1582. 


\section{Figure captions}

\section{Figure 1.}

Reported concentrations (ng/mL) and SDs in determination of PFOS in Serum A. The mean of the entries for each laboratory and the error bas for triplicate analysis by the laboratories are given by the filled symbols. The open symbols (o) are used when nondetects (NDs) or levels below DL were reported. The mean concentration is given by the solid line; the dotted lines indicate one or two times the RSD. The (n) refers to the number of mean concentrations submitted by the laboratories used for calculating the mean and \%RSDs.

Figure 2.

Reported concentrations (ng/mL) and SDs in determination of PFOA in Serum A.

Figure 3.

Reported concentrations $(\mathrm{ng} / \mathrm{mL})$ and SDs in determination of PFOS in Serum B.

Figure 4.

Reported concentrations $(\mathrm{ng} / \mathrm{mL})$ and SDs in determination of PFOA in Serum B

Figure 5.

Reported Concentrations (ng/mL) and SDs in determination of PFOS in Standard C.

Figure 6.

Reported Concentrations $(\mathrm{ng} / \mathrm{mL})$ and SDs in determination of PFOA in Standard C.

* All figure details as described in Figure caption 1.

Table 1.

Summary of the mean levels and \% RSD for Serum A, Serum B and Standard C in the second ILS on PFCs in human matrixes. 
Dear Editor,

Attached please find an edited version of the manuscript for the special Reach issue for the Journal of Chromatography.

We have implemented all changes and only the text is included, due to a misunderstanding the changes made by one of the reviewers were not correctly implemented in the 'track changes' format.

With best regards,

Gunilla Lindström

Man-Technology-Environment Research Centre (MTM)

Örebro University

SE-701 82 Örebr

Sweden

Tel. +4619301098

gunilla.lindstrom@nat.oru.se 


\section{Response Reviewer 2}

- Have the authors analyzed the results of the laboratories overall?

Lab 1 appears to be on the high side for PFOS and PFOA in the serum samples and the standard. This suggests a calibration error. Lab 14 appears to be on the low side. This suggests that the errors are not random, but rather systematic. Also, the z-score plots suggest this (e.g. lab 15 and 14 are on the low side and 5, 10 and 19 on the higher side). I think that the data could be analyzed for such relations using e.g. principle component analysis. Possible outcomes might support the statement that some labs need to check their calibration standards and procedures.

The focus of the paper is the overall quality of the data of PFC analysis and we decided not to focus on individual results. The general quality of the data in terms the RSDs between the participating laboratories covers both the systematic and random error of the analysis. We agree with the reviewer that this is interesting for (individual) laboratories but this might not be of more general interest for the reader of the J. of Chromatography. Including a more detailed individual discussion of the results by for example PLS or PCA would substantial prolong the article and is in our opinion somewhat outside the scope of the article.

- the z-score discussion (from line 314 onwards) is very brief, and no comparison was made to the results obtained in the 1st interlaboratory study (ref 2). I suggest to add this. Furthermore, one can add also the results of the other PFCs, e.g. by mentioning that (for example) $75 \%$ of the z-scores were satisfactory for PFHxS etc.

Other details:

Line 23: mass labelled instead of masslabelled 25, 26: PFCs instead of perfluorinated chemicals (abbreviation was already introduced)

Revised as suggested

27: matrices instead of matrixes

233: the ion trap cannot be used as MS/MS for the perfluoro sulfonates. Please clarify this, e.g. by LC-ESI-MS(/MS).

192 and 199: please add some details on the origin of the samples $A$ and $B$, like where they were sampled, whether or not from general population, occupationally exposed, red cross donation etc. This may be solved by referring to documents containing the information. Did the authors (or NIST) perform a test to demonstrate the homogeneity of both samples (e.g. between-lot homogeneity)?

Information on the samples from NIST 'certificate of analysis' is included, in addition reference to NIST is given where additional information can be obtained. 
278 and 302: to my

knowledge, the term "congeners"

is not appropriate here. I think that "homologue" would be more

correct. Please check this.

Revised as suggested

282 and also 296: the dataset was improved by removing 'obvious outliers', apparently according to a $>2$ sd criterium. Have the authors considered the use outlier tests (e.g. Grubbs) to check for outliers?

This may provide an improved basis for removal of outliers.

For the relatively small data set, the simple outlier test was used and found to be sufficient. Outlier detection and removal has a large influence on the analytical methods $\mathrm{RSD}$, in order to be able to compare the result which earlier studies and studies concerning other POPs where the same criteria where used we do not want to confuse the reader by introducing other outlier detection methods.

283: does $n$ refer to the number of measurements, or to the number of averages (each based on triplicate measurements)? Please clarify. Please clarify also if the open dot values (DL values) were included in the SD and RSD calculation.

Revised in the text and the Figure Caption. Note that this description was only included in Figure 1 and not for graphical identical Figures 2-6. We are not sure what the editorial policy of the J. of Chromotogr. is. Please advice.

292/293: please add a reference supporting the statement of 'background contamination levels'

A reference is included (ref 19 in the original manuscript)

294 to 296: something is not logic in the sentence "As an...are presented". Please restructure.

The sentence has been changed

316: please specify how the consensus value was calculated

This information has been included.

References: ref 7 and 18 are duplicates

Ref 18 has been deleted 
Figures: the figure explanations are very brief, and sometimes raise questions (which can easily avoided by adding more details).

Example 1: for all figures I would recommend to mention that all values are averages of triplicate determinations. Example 2: for

figure 2: provide information on the meaning of the open dots, and if they were included in the RSD calculation.

This information and more details are included in the Figure Caption for Figure 1. Figure 2-6 are graphical identical and the same information can be included, if needed for these figures.

Figure 4: please change PFOA to PFOS

Revised as suggested

Response Reviewer 1 (taken from comments entered in the manuscript)

We are thankful for the elaborate improvements of the language made directly on the document by reviewer 1 .

56 The target PFCs were PFBA, PFHxA, PFHpA, PFOA, PFNA, PFDA, PFUnA, PFDoA, PFBS, PFHXS, PFOS and PFOSA [These acronyms need to be initially defined in the body of the paper] .

The acronyms have been defined here.

125 for telomer alcohols, ToF [do the authors mean ToF/High resolution?] is the technique resulting in highest selectivity and sensitivity for anionic PFCs.

The definition of high resolution for current TOF instrument is somewhat different from traditional definitions ( $5 \%$ peak height, versus $50 \%$ peak height) so we consider this as TOF and not high resolution TOF.

127 quadrupole MS detection of anionic PFCs [7, 12]. [ I suggest that the chromatographic technique coupled to the detection technique be included for clarity]

This has been included

204 PFCs in the $n g / m L$ range [[We suggest that both Tables 1 and 2 be omitted.The content of the tables are presented in the text of the paper and do not add any additional information.]

Tables 1 and 2 have been deleted. 
A brief description of the sample intake [What is meant by "intake"?; is that the preparation or dilution step? Is this referring to the amout of sample prepared for analyses?], extraction, clean-up procedure, handling of final

This has been rewritten, sample preparation is a better description.

227 precipitation. Sample intakes intake [What is meant by "intake"?; is that the preparation or dilution step?] (from the reconstituted serums) were between $100 \mu \mathrm{L}$ and $1 \mathrm{~mL}$,

Here the amount of sample analyzed is a better description of the content.

274 (n) used in the calculations of all results.[ it is not clear where the minimums, maximums or medians are located in either the table or the figures]

This is a mistake which was not corrected; in an earlier version of the manuscript these values were included in Table 1 . This has now been corrected in the text.

277 [What is "satisfactory" - RSD $<50 \%$ ?].

For trace analysis at this level $50 \%$ is found to be satisfactory according to the Horowitz equation, for relatively new, non routine analysis.

The mean and RSD values for Figures 7 and 8 should be removed. They should not be presented with the z scores. It appears they have been inadvertently carried over from previous figures

This information has been included in the figures as a reference value for the z-scores, as explained in the text a 'floating' RSD was used to calculate the z-scores, depending on the results. This \%RSD is given in both Figure 7 and 8 , in addition to the number of results included after omitting obvious outliers. 


\section{Comments Journal Manager:}

When submitting your revision, can you please correct the following:

- insert an asterisk next to corresponding author's name

Ok.

- sections/subsections should be numbered, starting at 1. Introduction

Ok.

- Reference Section: Ref. 5, the correct abbreviation is $J$.

Chromatogr. B; Refs. 8 \& 10, the correct abbreviation is $J$.

Chromatogr. A; insert a full stop after each abbreviated word

Ok.

- figure captions should be typed together on a separate page and included at the end of the manuscript (after the references)

Ok, note that detailed information is only included for figure 1. Figure 2-6 are graphical identical and the same information can be included for these figure depending on the format of the article.

- each figure should be uploaded individually

Ok 